\title{
Comercio e infraestructura en la Comunidad Andina
}

\author{
Gina E. Acosta Rojas, Germán Calfat y Renato G. Flôres Jr.
}

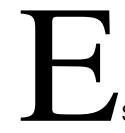

ste artículo examina el papel fundamental de la infraestructura en las modalidades de comercio de la Comunidad Andina, utilizando tres modelos de gravedad. El primero destaca la importancia de los acuerdos de comercio preferencial y de la proximidad geográfica. Los otros dos también abarcan ambos aspectos, pero se centran en la inclusión de la infraestructura en la ecuación de gravedad y comprueban que esta contribuye a reducir la "distancia" (entendida como los costos del transporte) entre las partes. Con los nuevos acuerdos comerciales, pierden importancia las fronteras y los acuerdos anteriores, el comercio será prácticamente libre y las corrientes bilaterales se definirán por los

Gina E. Acosta Rojas Investigadora asociada,

Instituto de Política y Gestión del Desarrollo, Universidad de Amberes

Germán Calfat

Profesor del mismo Instituto

• German.calfat@ua.ac.be

Renato G. Flôres Jr.

Profesor, Escuela de posgrado en economía, Fundación Getulio Vargas,

Río de Janeiro

•rfatwto@yahoo.com costos y la competitividad. Sin embargo, esto solo se logrará mejorando la infraestructura en todas las etapas de la cadena de producción y distribución. 


\section{Introducción}

En este trabajo se ofrecen nuevas pruebas de que el desarrollo de la infraestructura es fuente de integración y de competitividad, y se muestra el dinamismo de la función que cumple la infraestructura, tanto para explicar como para determinar las corrientes comerciales dentro y fuera de la Comunidad Andina.

El trabajo está estructurado de la siguiente manera. Las dos próximas secciones presentan el marco de análisis: la sección II reseña brevemente la evolución de lo que es hoy la Comunidad Andina, desde su iniciación en 1969 como Pacto Andino, centrándose en la consolidación del mercado interno y en el patrón de comercio. Para determinar si el Pacto y luego la Comunidad contribuyeron efectivamente a aumentar el comercio en la región, y a fin de captar los efectos de la proximidad geográfica en el comercio entre sus miembros, se aplica un modelo de gravedad aumentado de las corrientes bilaterales de comercio en todos los años correspondientes al período 1993-1999. En la sección III se presenta el primer modelo de gravedad. En la sección IV se examina el papel que desempeña la infraestructura en el comercio, pasando revista a las pruebas teóricas y estadísticas de que la ubicación y la dotación de recursos son concluyentes para determinar si los países optarán por aumentar el comercio mediante el mejoramiento de la infraestructura (con vistas a reducir los costos del transporte). A continuación, se da un vistazo a los medios de transporte que se utilizan en el comercio de la Comunidad Andina.

La sección $\mathrm{V}$, en que se evalúan plenamente los efectos del grado de desarrollo de la infraestructura, constituye el núcleo del trabajo. Más allá del modelo de gravedad tradicional, estimamos que los costos del transporte no son solamente una función de la distancia, sino también de la disponibilidad de medios adecuados, como ser caminos, energía y redes de telecomunicaciones. Estas variables se resumen en un índice que mide el grado de desarrollo de la infraestructura de los países en cuestión, modificando la variable distancia. Nuestro análisis contribuye a esclarecer el papel que desempeña la infraestructura y sus efectos en la importancia relativa de otras variables explicativas. A continuación, relacionamos los resultados con el nuevo concepto de desarrollo de la infraestructura en la región, cuya interacción con el espacio geográfico se considera un elemento clave de la integración y de la competitividad. Por último, en la sección VI se presentan las conclusiones del trabajo.

\section{II}

\section{Evolución de la Comunidad Andina}

Los inicios de lo que es hoy la Comunidad Andina se remontan a 1969, cuando un grupo de países suscribió el Acuerdo de Cartagena, conocido también como Pacto Andino, para establecer una unión aduanera en el plazo de 10 años.

Desde entonces, la integración andina ha atravesado por una serie de etapas diferentes a medida que el proyecto inicial de desarrollo hacia adentro basado en la sustitución de importaciones fue cediendo paso

$\square$ Renato Flôres agradece la acogida que le brindó el Instituto de Política y Gestión del Desarrollo, de la Universidad de Amberes, donde inició este trabajo en calidad de investigador invitado. a una iniciativa de mayor apertura regional. En junio de 1997, mediante el Protocolo de Trujillo, que modificó el Acuerdo de Cartagena, el grupo se convirtió en la Comunidad Andina. El Protocolo estableció un Consejo Presidencial Andino y un Consejo Andino de Ministros de Relaciones Exteriores, otorgándole a ambos un papel decisivo en el proceso de toma de decisiones. También fortaleció la organización interna del proceso de integración, entregando la gestión de todas las instituciones y mecanismos al Sistema Andino de Integración. En la actualidad, la Comunidad Andina es una organización regional con personalidad jurídica internacional. Últimamente han surgido algunas ten- 
siones entre los cinco países miembros — la República Bolivariana de Venezuela, Colombia, Ecuador, Perú y Bolivia- y Venezuela optó por adoptar un rumbo independiente. Al mismo tiempo, México solicitó su ingreso a la organización como miembro pleno. Sin embargo, ambos acontecimientos escapan al objetivo del presente trabajo.

A partir de 1987, para mantenerse al día con el proceso de liberalización que tenía lugar en América Latina, los países miembros empezaron a diseñar una nueva estrategia. La Zona de Libre Comercio, creada en 1992, evolucionó hasta convertirse en una unión aduanera imperfecta. En febrero de 1992, Colombia y la República Bolivariana de Venezuela eliminaron los aranceles y otras barreras al comercio recíproco. Bolivia se sumó a ellos en septiembre de 1992 y Ecuador lo hizo en enero de 1993, fecha en que entró en vigor la Zona de Libre Comercio entre los cuatro países. Perú suspendió transitoriamente el cumplimiento de sus obligaciones con arreglo al programa de liberalización, y a partir de 1992 empezó a negociar acuerdos bilaterales de comercio con cada uno de sus socios andinos y, en algunos casos, liberó parcialmente las corrientes de comercio recíprocas. Estos acuerdos bilaterales operaron hasta 1997, cuando se llegó a un acuerdo para la incorporación gradual de Perú a la Zona Andina de Libre Comercio (Decisión 414). Se eliminaron los aranceles que gravaban la mayoría de los productos hasta el año 2000, mientras que los "productos sensibles" restantes, incluidos los productos agrícolas, quedarían totalmente liberados en 2005.

En 1994, en virtud de la Decisión 370, se aprobó el Arancel Externo Común. Pero como siempre, aplicarlo ha sido difícil. Al momento de aprobarse esta Decisión, Bolivia estaba exenta y, como se dijo, Perú no participó en el proceso. Una vez más, los primeros en aplicarlo fueron Colombia y la República Bolivariana de Venezuela en 1994, seguidos por Ecuador en 1995. El Arancel Externo Común andino depende del grado de elaboración de los productos y se aplica una tasa de 5\% a las materias primas y a los insumos industriales, de $10 \%$ y $15 \%$ a los insumos intermedios y a los bienes de capital, respectivamente, y de $20 \%$ a los bienes de consumo final. En promedio, el Arancel Externo Común es de 13,6\%, con un máximo de $20 \%$. Bolivia y Perú se están incorporando gradualmente a la unión aduanera vigente para Ecuador, Colombia y la República Bolivariana de Venezuela. Se esperaba la plena adopción en el 2005.

La Comunidad Andina ha abordado la mayoría de los temas de discusión más nuevos en materia de comercio, tales como las inversiones, la política de competencia, los servicios y los derechos de propiedad intelectual, y ha adoptando políticas comunes en la mayoría de estos campos; ${ }^{1}$ además, se ha preocupado del problema de la infraestructura, que es materia del presente trabajo. También está consciente de que su principal objetivo es desarrollar una política externa común, lo que involucra la participación conjunta de todos sus miembros en la Organización Mundial de Comercio (OMC) y en las negociaciones relacionadas con los acuerdos regionales.

En 2004 los países andinos representaban un mercado de más de 121 millones de personas, que viven en una superficie de 4.700 .000 kilómetros cuadrados. Ese año, el PIB conjunto se elevó a 317 mil millones de dólares. Los principales mercados de exportación son Estados Unidos, la Unión Europea (UE) y la propia Comunidad.

La liberalización del mercado interno ha tenido importantes consecuencias para el comercio entre los países miembros. Las corrientes comerciales han alcanzado niveles sin precedentes, y el comercio intrarregional está aumentando más rápidamente que el intercambio con el resto del mundo. Tras un escaso o bajo crecimiento en la década de 1980, en 1989 el comercio intraandino repuntó y a partir de 1990 comenzó a aumentar sostenidamente. A fines del 2004, las exportaciones intraandinas se elevaron a 7.400 millones de dólares, esto es, casi triplicaron el valor alcanzado en 1992. Asimismo, es importante señalar que el comercio andino con el resto del mundo también ha crecido. Las importaciones y exportaciones desde países de la Comunidad y hacia otros no pertenecientes a ella han aumentado en forma sostenida desde que se reactivó el acuerdo a principios de los años 1990.

Pese al compromiso de establecer un mercado común, la Comunidad — como se dijo— aún funciona como una unión aduanera incompleta, ya que tanto el Arancel Externo Común como la Zona de Libre Comercio tienen una serie de excepciones.

\footnotetext{
${ }^{1}$ A manera de ejemplo, la Decisión 291 reemplazó a la Decisión 24, que limitaba las actividades relacionadas con la inversión extranjera directa, otorgando a los inversionistas extranjeros los mismos derechos y obligaciones de los nacionales y eliminando todas las barreras a las remesas de capital y de utilidades. La Decisión 344 otorgó derechos de patente a los productos farmacéuticos y la Decisión 351 se ocupó de los derechos de autor.
} 


\section{III}

\section{Un primer modelo de gravedad}

Para establecer un marco a partir del cual analizar el crecimiento del comercio entre los países andinos, se construyó la siguiente ecuación de gravedad:

$$
\begin{aligned}
\ln M_{i j}=\beta_{0}+ & \beta_{1} \ln Y_{i} Y_{j}+\beta_{2} \sqrt{D_{i j}}+\beta_{3} A C P \\
& +\beta_{4} \text { Border }+e_{i j}
\end{aligned}
$$

en que $M_{i j}$ representa el valor de las importaciones del país $i$ desde el país $j$, mientras que $Y_{i} Y_{j}$ es el producto interno bruto (PIB) multiplicado de ambos países como sustituto del tamaño, $D_{i j}$ la distancia entre el país $i$ y el país $j$ para captar los costos comerciales, $A C P$ una variable ficticia para medir los efectos de la integración en el comercio de los países miembros, cuyo valor es igual a 1 cuando ambos países pertenecen a la Comunidad Andina y 0 en caso contrario, y Border (frontera) una variable ficticia para medir los efectos de la proximidad, cuyo valor es igual a 1 cuando los países tienen una frontera común. ${ }^{2}$

Se analizó el período 1993-1999, dado que la integración cobró impulso tras la firma del acuerdo de libre comercio en 1992, y nos propusimos determinar la importancia y el valor de sus efectos en el comercio intrarregional. En la ecuación (1), los países que están a la izquierda son los cinco miembros de la Comunidad Andina y los que se encuentran a la derecha, los socios, vale decir, sus proveedores o exportadores. La elección de los socios se basó en la existencia de comercio bilateral con los países miembros.

La información sobre los flujos comerciales, en millones de dólares corrientes, se obtuvo del Fondo Monetario Internacional (FMI, 2001). La información sobre el PIB, en dólares corrientes, corresponde a la base de datos sobre el desarrollo mundial del Banco Mundial, ${ }^{3}$ mientras que la distancia entre las capitales, en kilómetros, se sacó de la página web de Haveman. ${ }^{4}$

\footnotetext{
${ }^{2}$ Frankel (1997) utilizó modelos de gravedad para demostrar que la regionalización puede explicarse por la proximidad geográfica y los acuerdos de comercio preferenciales. Krugman (1991) expresó el papel desempeñado por la proximidad geográfica en el proceso de regionalización, y a partir de entonces se han utilizado variables dummy para simular y analizar esos efectos. Anderson y van Wincoop (2003) ofrecen un respaldo teórico mejor y más reciente para todo esto.

${ }^{3}$ www.worldbank.org/research/growth/GDNdata.html.

${ }^{4}$ www.haveman.org.
}

Basándose en la ecuación (1), se corrieron regresiones individuales para cada año. Antes de ello, se realizó un análisis descriptivo de los datos. Esto condujo a transformar las importaciones y el PIB por logaritmo natural y la distancia mediante su raíz cuadrada. Se utilizó el método de mínimos cuadrados ordinarios, considerando los datos de las importaciones modificados como variables dependientes. Cada año se eliminó una serie de países de Asia y África que no tenían intercambio con la Comunidad Andina.

El cuadro 1 muestra los resultados, en coeficientes estandarizados, junto al valor $\mathrm{R}^{2}$ de cada regresión y la significancia de los coeficientes. La ecuación de gravedad sirve adecuadamente para explicar el comercio bilateral entre los países andinos y sus socios respectivos. El ajuste general de la regresión es satisfactorio, puesto que los valores de $\mathrm{R}^{2}$ son superiores a 0,70 . En todos los casos, las variables independientes tuvieron el signo esperado y resultaron estadísticamente significativas de acuerdo con las pruebas de $\mathrm{F}$ y $\mathrm{t}$.

El efecto de la multiplicación del PIB de los países es positivo y estadísticamente significativo, y fluctúa entre 0,862 y 0,901 . Estos valores coinciden con los encontrados por Frankel (1997) y Echavarría (1998) para los períodos 1965-1980 y 1986-1995, respectivamente, aunque son un poco más altos, porque en la actualidad el tamaño influye más en el comercio y porque, como es de suponer, en cada análisis se eligieron contrapartes diferentes. Los coeficientes cumplen con la hipótesis de que el tamaño de la economía aumenta a la par con el comercio y, en el caso de los países andinos, influye marcadamente en el comercio.

Los coeficientes de distancia son negativos, estadísticamente significativos y su valor fluctúa entre $-0,443$ y $-0,345$. Sin embargo, la distancia influye menos que el PIB. El valor y el signo de los coeficientes de distancia también son similares a los encontrados por Frankel (1997) y Echavarría (1998). Ambos autores trabajaron con un período anterior a aquel en que se liberalizaron los servicios de transporte y se redujeron los costos, de manera que en la mayoría de los casos sus coeficientes son superiores a los obtenidos en este trabajo, realizado cuando el efecto distancia ya había disminuido.

Los coeficientes de la variable ficticia del acuerdo sobre comercio preferencial fluctúan entre 0,101 y 
(En coeficientes estandarizados)

\begin{tabular}{|c|c|c|c|c|c|c|c|}
\hline & 1993 & 1994 & 1995 & 1996 & 1997 & 1998 & 1999 \\
\hline$Y_{i} Y_{j}$ & $0,897 *$ & $0,862 *$ & $0,896^{*}$ & $0,882^{*}$ & $0,901 *$ & $0,867 *$ & $0,865^{*}$ \\
\hline$D_{i j}$ & $-0,435^{*}$ & $-0,403 *$ & $-0,443^{*}$ & $-0,413^{*}$ & $-0,377^{*}$ & $-0,347^{*}$ & $-0,345^{*}$ \\
\hline D Border & $0,200 *$ & $0,161 *$ & $0,129 *$ & $0,124 *$ & $0,127 *$ & $0,116 *$ & $0,139 *$ \\
\hline $\mathrm{N}^{\mathrm{o}}$ observaciones & 141 & 243 & 240 & 255 & 247 & 261 & 235 \\
\hline $\mathrm{R}^{2}$ & 0,82 & 0,722 & 0,755 & 0,752 & 0,780 & 0,714 & 0,769 \\
\hline
\end{tabular}

Fuente: estimaciones de los autores.

* Significativo al $5 \%$.

0,160. Su significancia estadística (valores p) mejora a partir de 1995 y evolucionan en forma positiva, aunque a bajos niveles (gráfico 1). Cabe recordar que la Zona de Libre Comercio solo entró en vigor en 1993 y que Perú se mantuvo fuera del Pacto hasta 1997. Además, el elevado número de excepciones en que se aplican normas distintas contribuye a reducir el grado de influencia del acuerdo. Se prevé que los efectos del Pacto serán mayores a medida que todas las partes apliquen más uniformemente las normas. La evolución positiva de los coeficientes y el hecho de que aumente su significancia indica que, salvo en 1999, los países miembros están comerciando cada vez más entre sí. Ese año se produjeron numerosas perturbaciones económicas y políticas, tales como la crisis macroeconómica y bancaria en Ecuador, los problemas políticos en Perú que condujeron a la huida del presidente Fujimori y las inundaciones en la República Bolivariana de Venezuela. En general, los resultados de nuestras pruebas muestran que el Pacto y la Zona de Libre Comercio influyeron favorablemente en el comercio en los países miembros.

La variable ficticia correspondiente a la proximidad se utiliza para calcular si las fronteras comunes, que posibilitan el comercio fronterizo, efectivamente contribuyen a aumentar las corrientes comerciales. Los coeficientes de esta variable son positivos y estadísticamente significativos, aunque sus valores son bajos y evolucionan
GRÁFICO 1

Evolución de la variable ficticia ACP

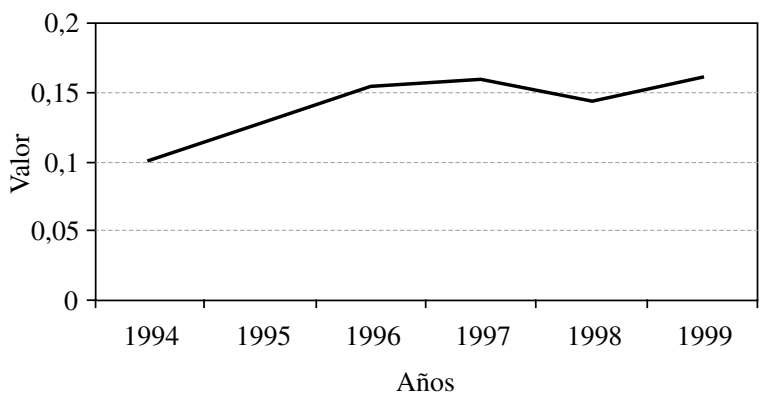

Fuente: estimaciones de los autores basadas en el cuadro 1 .

más bien a la baja. Los valores positivos confirman que los países que tienen una frontera común comercian más entre sí, pero el hecho de que el valor sea bajo y de que no muestren una tendencia positiva indica que estas economías son relativamente pequeñas y posiblemente comercien más con otras de mayor tamaño, pese a que estén geográficamente distantes. Cabe mencionar que los países suelen no comerciar más con sus vecinos por la falta de una infraestructura de transportes adecuada o porque su ubicación geográfica es compleja, como en el caso de la cordillera de Los Andes, elemento que puede elevar apreciablemente los costos. 


\section{IV}

\section{Comercio e infraestructura en la}

\section{Comunidad Andina}

\section{Comercio, infraestructura e integración regional}

Desde que en 1991 Krugman recordó la importancia de los factores geográficos para el comercio, varios autores tales como Hummels (1998) han tratado de medir los efectos de la distancia y el papel que desempeña la infraestructura en un modelo de comercio bilateral. En algunos estudios empíricos, como el de Porojan (2000), se utilizaron datos sobre las inversiones como sustituto de la infraestructura. Sin embargo, como lo advirtieron Summers y Heston (1991), cuando se usa este tipo de datos para calcular el capital invertido en infraestructura pueden surgir algunos problemas. Es posible que la eficacia de la misma corriente de inversión varíe, según el país de que se trate, debido a diferencias en cuanto a la eficiencia del sector público y a los precios del capital destinado a infraestructura.

Bougheas, Demetriades y Morgenroth (1999) trataron de examinar el papel que desempeña la infraestructura en un modelo de comercio bilateral y en el costo del transporte. Sus conclusiones indican que en el caso de dos países en que la inversión en infraestructura es óptima existe una relación directamente proporcional entre la dotación de infraestructura y el volumen de comercio. En consecuencia, las variaciones del costo del transporte entre los distintos países podrían explicar las diferencias en su capacidad de competir en los mercados internacionales. Además, es probable que las diferencias en cuanto a la cantidad y calidad de la infraestructura expliquen las diferencias en los costos del transporte, y, en consecuencia, las diferencias en materia de competitividad. Como resultado de lo anterior, disminuyendo los costos y mejorando la calidad de los sistemas de transporte aumenta el acceso a los mercados internacionales y se promueve el incremento del comercio.

Hay datos que vinculan categóricamente el mejoramiento de los servicios de transporte y de la infraestructura en general con el mejor comportamiento de las exportaciones. Hummels (1999) calcula que los exportadores que pagan $1 \%$ menos por los fletes aumentarán su participación en el mercado entre 5 y $8 \%$.
Limão y Venables (2001) calcularon que la elasticidad de los flujos comerciales respecto del factor costo del comercio es de aproximadamente -3 , y estudiaron en qué medida los costos del transporte dependen de la ubicación geográfica y de la infraestructura. Concluyeron que las diferencias en materia de infraestructura explican $40 \%$ de la variación de los costos del transporte en el caso de los países costeros y hasta $60 \%$ en aquellos sin litoral. Además, Wilson (2003) demostró que en los países del Foro de Cooperación Económica Asia-Pacífico (APEC) hay grandes diferencias en cuanto a la calidad de la infraestructura de transportes y al nivel de los servicios logísticos y comerciales, y que estas diferencias explican la brecha que acusan los resultados de sus actividades comerciales. El estudio concluye que el mejoramiento de la infraestructura de transporte y de servicios aumentará sustancialmente el comercio en los países que van a la zaga en esta materia.

Martinez-Zarzoso y Nowak-Lehmann (2002) estudiaron el papel que desempeñan las diferencias en materia de actividad económica y la distancia en algunas exportaciones sectoriales del Mercosur a la UE. Sus conclusiones revelan que existe una relación inversamente proporcional entre la distancia geográfica, definida como la distancia física expresada en kilómetros entre las capitales modificada por un índice de la infraestructura, que tiene efectos adversos en el comercio. La distancia aumenta los costos del transporte, pero estos pueden reducirse con una mejor infraestructura.

Los costos reales del comercio, incluidos el transporte y el costo de realizar negocios en el plano internacional, son factores decisivos en la capacidad de un país determinado de participar en la economía mundial. Como lo señalaron Limão y Venables (2001), la lejanía y las deficiencias en materia de infraestructura de transportes y comunicaciones contribuyen a aislar a los países y limitan su capacidad de participar en las cadenas de producción internacionales. Cualquier estrategia orientada a aumentar la competitividad internacional de una región debe contemplar el mejoramiento de los canales que facilitan el intercambio de bienes, servicios y personas.

Por lo que respecta a la integración regional, como lo señala el BID (2000), la interacción geográfica genera 
flujos que no necesariamente circulan libremente, sino a través de redes de infraestructura. Estas redes proporcionan el respaldo material por conducto del cual circulan las corrientes de comercio: para asegurar que influyan positivamente en la integración y el desarrollo se requieren un marco jurídico e institucional adecuado así como servicios eficaces en materia de infraestructura. Además, como - al igual que el proceso de integración- son un bien público regional (BID, 2004), para que adquieran plenamente su carácter de tal es preciso que todos los países involucrados actúen conjunta y coordinadamente.

\section{El comercio en la Comunidad Andina según el medio de transporte utilizado}

Para determinar las variables que afectan los costos del transporte en el comercio intracomunitario, es importante examinar los medios de transporte utilizados. El cuadro 2 ofrece información sobre el comercio de la Comunidad Andina según el medio de transporte de que se trate. Entre 1997 y 1999, la mayor parte de las exportaciones intracomunitarias se realizaron por carretera; en realidad, casi $49 \%$ del valor total del comercio. El transporte marítimo ocupó el segundo lugar, con casi 38\% del valor comerciado, mientras que el transporte aéreo se situó tercero, con aproximadamente $8 \%$ del total.

En 1997, el transporte terrestre fue el principal sistema utilizado por Bolivia, Colombia, Ecuador y la República Bolivariana de Venezuela. En 1998, aumentó la participación del transporte marítimo en Ecuador, y en 1999 en Venezuela. Entre 1997 y 1999, 48\% de las exportaciones venezolanas hacia otros países andinos se transportaron por carretera y $39 \%$ por vía marítima; por su parte, $62 \%$ de las importaciones de sus socios andinos se transportó por tierra y $29 \%$ de ellas por mar. Así

CUADRO 2

Comunidad Andina: exportaciones intracomunitarias por medio de transporte, 1997-1999

(En porcentajes de su valor)

\begin{tabular}{lrrr}
\hline Medio de transporte & 1997 & 1998 & 1999 \\
\hline Terrestre & 49,5 & 51,0 & 45,7 \\
Marítimo & 38,5 & 36,5 & 39,9 \\
Ferroviario & 0,5 & 0,3 & 0,7 \\
Aéreo & 5,7 & 8,7 & 9,2 \\
Multimodal & 0,1 & 0,0 & 0,0 \\
Fluvial & 5,6 & 2,9 & 4,4 \\
Otros & 0,0 & 0,6 & 0,1 \\
\hline
\end{tabular}

Fuente: www.comunidadandina.org también, en el mismo período 55\% de las exportaciones colombianas se despachó por carretera y $35 \%$ por vía marítima, mientras que $60 \%$ de las importaciones llegó por tierra y $33 \%$ por mar.

El transporte marítimo es utilizado principalmente por Perú para todas sus entregas y por otros miembros de la Comunidad cuando el intercambio tiene lugar con países que no tienen una frontera común, lo que hace que el transporte interior sea caro y lento. Este medio de transporte es el que utilizan tradicionalmente los países andinos cuando el intercambio tiene lugar con socios distantes, como Estados Unidos y la UE, con lo cual ocupa el segundo lugar en el movimiento de productos desde y hacia la región andina. En todo caso, cabe tener en cuenta que en los casos en que se utiliza el transporte marítimo casi siempre se requiere recorrer una distancia adicional, ya sea por carretera o por ferrocarril, tanto en el lugar de origen como en el de destino. Bolivia es el principal ejemplo al respecto, debido a que es un país sin litoral. Por lo general, tanto para las exportaciones como para las importaciones hacia y desde países no limítrofes, combina el despacho a un puerto chileno con el transporte terrestre (Comunidad Andina, s/f).

En general, los miembros de la Comunidad no utilizan medios de transporte fluvial y lacustre debido a que en las zonas en que ello sería factible no hay vías adecuadas. Además, los sectores comerciales de cada país a menudo se encuentran en lugares en que solo es posible utilizar medios de transporte terrestre o marítimo.

El transporte aéreo es relativamente limitado: es más rápido enviar las mercancías por carretera, especialmente si el comercio tiene lugar entre miembros de la Comunidad que poseen una frontera común. Además, cuando se trata de cruzar la frontera el medio más expedito es el transporte terrestre. ${ }^{5} \mathrm{El}$ intercambio por vía área con contrapartes situadas fuera de la región andina es limitado y se reduce a productos altamente perecederos.

Entre 1997 y 1999, el comercio fronterizo de los miembros de la Comunidad representó $98 \%$ del comercio intracomunitario por carretera y $49 \%$ del total. Por consiguiente, entre los miembros que no comparten fronteras el comercio por este medio fue escaso. Como puede apreciarse en el cuadro 3, entre Colombia y la República Bolivariana de Venezuela el comercio fronterizo por carretera es muy significativo y representa alrededor de $66 \%$ del total de la región. El comercio

\footnotetext{
${ }^{5}$ Intercambio personal con la empresa ZaiMella del Ecuador S.A., que realiza actividades relacionadas con la exportación y la importación en la mayoría de los países miembros de la Comunidad Andina.
} 
(En millones de dólares)

\begin{tabular}{|c|c|c|c|c|c|}
\hline País fronterizo de destino & 1997 & 1998 & 1999 & 1997-1999 & $\%$ \\
\hline Bolivia a Perú & 143 & 120 & 68 & 331 & 4,50 \\
\hline Colombia a Ecuador & 353 & 360 & 198 & 911 & 12,38 \\
\hline Colombia a Perú & 7 & 2 & 0 & 9 & 0,12 \\
\hline Colombia a Venezuela (R.B.) & 802 & 847 & 688 & 2337 & 31,77 \\
\hline Ecuador a Colombia & 336 & 269 & 207 & 812 & 11,04 \\
\hline Ecuador a Perú & 23 & 11 & 13 & 47 & 0,64 \\
\hline Perú a Bolivia & 92 & 91 & 84 & 267 & 3,63 \\
\hline Perú a Colombia & 3 & 1 & 2 & 6 & 0,08 \\
\hline Perú a Ecuador & 64 & 34 & 14 & 112 & 1,52 \\
\hline Venezuela (Rep. Bol. de) a Colombia & 982 & 1073 & 470 & 2525 & 34,32 \\
\hline Total & 2805 & 2807 & 1744 & 7357 & 100,00 \\
\hline
\end{tabular}

Fuente: www.comunidadandina.org

entre Colombia y Ecuador se sitúa en segundo lugar, con poco más de $23 \%$ del total, y el de Bolivia y Perú ocupa el tercer lugar (8\%), aunque en este mismo período casi la mitad del comercio total entre ambos países se realizó por carretera. La cifra más baja corresponde al comercio entre Ecuador y Perú, con solo $2 \%$ del total.

A fines de los años 1980, la falta de infraestructura y la escasa proyección del Pacto Andino hizo que el hecho de compartir una frontera común fuera extremadamente importante para el comercio entre sus miembros. Este tenía lugar en la frontera y había poco interés en comerciar a distancia, ya que los servicios logísticos y de transporte eran escasos y costosos. Sin duda alguna, en esa época la distancia desempeñó un papel muy importante y las fronteras determinaron quiénes debían ser la contraparte natural en el intercambio. Como lo demuestran los coeficientes de la variable ficticia en el modelo (1), en el decenio de 1990 se redujo apreciablemente la importancia del comercio fronterizo.

\section{V}

\section{Evaluación del efecto infraestructura}

\section{Características del modelo e información al respecto}

Los resultados del modelo (1) pusieron de relieve que el tamaño de la economía (PIB) es tal vez la variable más importante cuando se trata de elegir un socio comercial y que en materia de costos la distancia es un factor decisivo. Sin embargo, el hecho de que el valor absoluto de los coeficientes de distancia disminuyera a lo largo del período indica que, además de la distancia física, es posible que en la región andina haya otros factores que influyen en el costo del transporte (y, por lo tanto, en el comercio). En efecto, como en el período estudiado la economía de las contrapartes bilaterales no varió demasiado, no se modificaron las fronteras ni varió la estructura básica del Pacto, habría que analizar más a fondo la variable del costo del transporte y todos los elementos conexos.

A partir de los estudios descritos en la sección IV, apartado 1, para determinar la importancia de la infraestructura en el comercio construimos un modelo de gravedad aumentado en que la distancia física se modifica mediante un índice de infraestructura, es decir, una distancia geográfica centrada en la relación recíproca entre el aspecto geográfico y la infraestructura. Los costos del transporte se convierten no solo en una función de la distancia, sino también de la dotación de infraestructura pública, como ser carreteras, ferrocarriles y redes de energía y telecomunicaciones. Estos aspectos de la infraestructura pública se resumen en un índice que mide el grado de desarrollo de la infraestructura de los países, modificando la variable distancia. 
Reformulando la ecuación (1), el comercio bilateral se expresa como:

$$
\begin{aligned}
\ln M_{i j}=\beta_{0}+ & \beta_{1} \ln Y_{i} Y_{j}+\beta_{2} G e o D_{i j}+\beta_{3} A C P \\
& +\beta_{4} \text { Border }+e_{i j}
\end{aligned}
$$

en que $M_{i j}, Y_{i} Y_{j}, A C P$ y Border son iguales que en (1), y $G e o D_{i j}$ representa la distancia entre el país $i$ y el país $j$ modificada por el índice de infraestructura.

En el análisis se utiliza un corte transversal del período 1985-1995. ${ }^{6}$ Una vez más, los países estudiados son los cinco miembros de la Comunidad Andina, las contrapartes se eligieron de acuerdo con el volumen de comercio con los países andinos y la disponibilidad de información respecto de su dotación de infraestructura. Conservando las variables ficticias que representan los efectos del Pacto Andino y de las fronteras, al agregar la infraestructura el análisis sigue captando la importancia del acuerdo de comercio preferencial y del hecho de compartir fronteras.

La información sobre las corrientes de comercio bilaterales y el PIB se obtuvo de las mismas fuentes anteriores. La variable distancia geográfica es similar a la utilizada por Martinez-Zarzoso y Nowak-Lehmann (2002) y Limão y Venables (2001). Se define como la distancia física entre las capitales de los socios comerciales (obtenida en la forma anterior), dividida por la suma del índice de infraestructura de ambos países. El índice se basó en cinco variables, a saber, kilómetros de carreteras, carreteras pavimentadas y vías férreas, troncales telefónicas y kilowatts de capacidad de generación eléctrica, y se explican en el apéndice.

Las cifras anuales sobre la existencia de infraestructura física del país informante y de sus socios comerciales en el período 1985-1995 se obtuvieron de la base de datos de David Canning, correspondiente a 1998. ${ }^{7}$ Los datos que ofrece Canning son de dos tipos: antecedentes brutos con un mínimo de manipulación y prácticamente tal como aparecen en las fuentes originales y datos elaborados que incluyen algún tipo de interpolación (por ejemplo, el supuesto de que en el período en cuestión se produjo un crecimiento exponencial). Como lo recomienda ese autor, se utilizaron los datos elaborados, debido a su coherencia intertemporal para calcular el índice para el trabajo empírico. Los datos

\footnotetext{
${ }^{6}$ La diferencia de tiempo en relación al modelo (1) se ajustó de acuerdo con los datos sobre dotación de infraestructura.

${ }^{7}$ Data Base of World Infrastructure Stocks, disponible en www.worldbank. org/html/dec/Publications/workpapers/WPS1900series/wps1929.
}

sobre población y región del país para normalizar las existencias de infraestructura se obtuvieron de una base de datos ${ }^{8}$ y de la página web de Country Watch, respectivamente. ${ }^{9}$

\section{Resultados empíricos}

En el caso del modelo (2) se corrieron regresiones utilizando mínimos cuadrados ordinarios para cada año, con el logaritmo natural de las importaciones de los miembros del acuerdo como variable dependiente. Una vez más, se excluyeron de la muestra varios países de Asia y África que no participaron en comercio bilateral con la Comunidad Andina.

Los resultados (en coeficientes estandarizados) de cada regresión figuran en el cuadro 4 . En el período $1985-1991$, los valores $R^{2}$ fluctuaron entre 0,653 y 0,735 , y de 1992 a 1995 en todos los casos fueron superiores a 0,712. Por tanto, la ecuación de gravedad explica adecuadamente el comercio bilateral de los cinco miembros del Pacto Andino, especialmente en la segunda parte del período, lo que indica la mayor aplicación e importancia del acuerdo sobre comercio preferencial.

Nuevamente, la variable más importante es el tamaño de la economía, lo que no solo confirma los hallazgos ya presentados, sino que coincide con los de la mayoría de los estudios empíricos. Sin embargo, cabe destacar que si en la ecuación se incluye la infraestructura, el efecto del tamaño de la economía (el PIB multiplicado de cada par de países) es menor. Si bien es cierto que el poder de compra de la contraparte es el primer requisito para que haya comercio, el hecho de que en este modelo el PIB tenga menos incidencia confirma que la dotación de infraestructura tanto del miembro de la Comunidad como de su contraparte comercial reducen la distancia entre ellos. De hecho, al disminuir el costo del transporte y, en consecuencia, el precio de los productos transados, estos se tornan más accesibles y se acorta la distancia económica entre los mercados. A lo largo del período estudiado, los coeficientes del PIB son estadísticamente significativos, positivos y no varían de manera importante de un año a otro. Fluctúan entre 0,718 y 0,791 y se asemejan a los encontrados por Echavarría (1998) y Frankel (1997) en otros estudios empíricos sobre la Comunidad Andina, aunque de todas formas son superiores.

\footnotetext{
${ }^{8}$ Global Development Network Growth Database.

${ }_{9}^{9}$ Disponible en www.worldbank.org/research/growth/GDNdata.html; www.countrywatch.com.
} 
Pacto Andino, 1985-1995: Primer modelo de gravedad incluida la infraestructura. Resultados empíricos

(En coeficientes estandarizados)

\begin{tabular}{|c|c|c|c|c|c|c|}
\hline Año & En $Y_{i} Y_{j}$ & En distancia geográfica & Variable ficticia ACP & Variable ficticia border (frontera) & $\mathrm{R}^{2}$ & $\mathrm{~N}^{\mathrm{o}}$ \\
\hline 1985 & $0,744^{*}$ & $-0,252 *$ & 0,007 & $0,410^{*}$ & 0,677 & 125 \\
\hline 1986 & $0,729 *$ & $-0,250^{*}$ & 0,021 & $0,384^{*}$ & 0,664 & 129 \\
\hline 1987 & $0,743^{*}$ & $-0,243^{*}$ & 0,032 & $0,374 *$ & 0,666 & 131 \\
\hline 1988 & $0,780^{*}$ & $-0,211^{*}$ & 0,041 & $0,390^{*}$ & 0,717 & 134 \\
\hline 1989 & $0,727 *$ & $-0,244^{*}$ & 0,080 & $0,371 *$ & 0,653 & 133 \\
\hline 1990 & $0,773^{*}$ & $-0,206^{*}$ & $0,170^{*}$ & $0,386^{*}$ & 0,692 & 140 \\
\hline 1991 & $0,798 *$ & $-0,228 *$ & $0,243 *$ & $0,349 *$ & 0,735 & 132 \\
\hline 1992 & $0,791 *$ & $-0,256^{*}$ & $0,159 *$ & $0,371 *$ & 0,757 & 135 \\
\hline 1993 & $0,786^{*}$ & $-0,245^{*}$ & $0,197 *$ & $0,339 *$ & 0,777 & 143 \\
\hline 1994 & $0,750^{*}$ & $-0,256^{*}$ & $0,227 *$ & $0,307^{*}$ & 0,728 & 146 \\
\hline 1995 & $0,718^{*}$ & $-0,293 *$ & $0,237^{*}$ & $0,264^{*}$ & 0,712 & 151 \\
\hline
\end{tabular}

Fuente: estimaciones de los autores.

* Significativo al $5 \%$.

La variable ficticia del Pacto Andino no resultó significativa antes de 1990. Hasta entonces, las políticas de sustitución de importaciones y de regionalismo hacia adentro indicaron que existía un acuerdo plagado de excepciones y carente de funciones operativas, debido a que los miembros no cumplían cabalmente con sus requisitos: todos aplicaban aranceles altos y había numerosas barreras no arancelarias. Los resultados de la variable ficticia del Pacto confirman que el acuerdo de comercio preferencial no ejerció influencia alguna en el intercambio entre sus miembros antes de que las reformas orientadas al mercado sentaran las bases para dar impulso a los esfuerzos de integración. Por desgracia, pese al establecimiento de la Zona de Libre Comercio, en 1992 la República Bolivariana de Venezuela congeló sus relaciones diplomáticas con Perú a raíz de la crisis en este país. A consecuencia de ello, ese año Perú suspendió transitoriamente el cumplimiento de sus obligaciones de acuerdo con el programa de liberalización. Estos sucesos le restaron a la Comunidad el respaldo político necesario y provocaron una caída del comercio entre sus miembros, como lo confirma la baja del coeficiente de la variable, por mucho que continuara siendo significativa. A partir de entonces los valores han acusado una tendencia positiva que indica que el acuerdo se ha cumplido sostenidamente.

Los nuevos valores de la variable ficticia para el Pacto también son superiores a los que se obtuvieron cuando el modelo no incluyó la dotación de infraestructura. ${ }^{10}$

10 Tanto en este apartado como en el siguiente, cuando se realizan afirmaciones sobre el tamaño relativo de los mismos coeficientes en
Al incluirla no solo se atenuó el efecto de la distancia, sino que se fortaleció el papel desempeñado por el acuerdo de comercio preferencial. La combinación de la infraestructura adecuada y el fortalecimiento permanente de la integración regional en distintos frentes, sin duda, seguirá influyendo positivamente en el comercio intracomunitario.

La variable ficticia correspondiente a las fronteras no solo arrojó resultados estadísticamente significativos a lo largo de todo el período, sino que adquirió mayor importancia como factor determinante del comercio. Al igual que en el caso de la variable ficticia anterior, al incluir la infraestructura aumenta la importancia de las fronteras. En los años pertinentes (1993-1995), todos los coeficientes fueron superiores a 0,620 y prácticamente duplicaron los obtenidos con el modelo (1). La importancia de las fronteras en el comercio de la Comunidad Andina coincide con el hecho ya mencionado de que casi $50 \%$ del comercio dentro de las regiones se realiza por carretera y $98 \%$ del transporte que se realiza por carretera tiene lugar en la frontera. Sin embargo, se mantiene una de las características más importantes de los resultados de esta variable en el modelo (1), es decir, la tendencia decreciente de los coeficientes. Hasta 1992 los valores de los coeficientes fueron superiores, lo que indica que compartir una frontera era mucho más importante que haber suscrito un acuerdo comercial. Pese a existir, estos

las distintas regresiones (sea en modelos diferentes para el mismo año o en el mismo modelo en años diferentes) se realizaron las pruebas de significancia del caso. A fin de simplificar el texto, no incluimos los resultados, pero ellos pueden consultarse a los autores. 
no se cumplían cabalmente. Estos valores más altos también reflejan dos aspectos adicionales: la existencia de una infraestructura más deficiente y el mayor costo resultante del transporte de la mercancía por un medio de transporte distinto del terrestre. A partir de 1992, cuando entró en vigor la Zona de Libre Comercio y los costos del transporte marítimo disminuyeron a cifras razonables en función del tránsito y la frecuencia de los viajes, la importancia de compartir fronteras se redujo y sus niveles se aproximaron a los coeficientes correspondientes a la distancia geográfica y la variable ficticia del Pacto.

Los coeficientes de la distancia geográfica fueron estadísticamente significativos y de signo negativo, confirmando así que, incluso en la forma en que se representan en el modelo, los costos del transporte reducen el comercio. Los resultados también respaldan el marco teórico, ya mencionado, en el sentido de que la dotación de infraestructura reduce las distancias bilaterales. Los coeficientes de la distancia geográfica equivalen aproximadamente a la mitad de los obtenidos cuando los costos del transporte se sustituyeron únicamente por la distancia física. ${ }^{11}$ A partir de 1990 evolucionan positivamente. Esta evolución es contraria a la encontrada cuando solo se utilizó la distancia física y revela que la importancia del término "distancia" aumenta a medida que aumenta la competencia por servicios de

\footnotetext{
${ }^{11}$ El lector debería tener en cuenta que en el modelo (1) se utilizó la raíz cuadrada de la distancia, aunque ello no invalida los comentarios incluidos en este párrafo.
}

transporte y se utilizan medios nuevos y mejores de despachar mercancías, que se tornó más flexible y que su reducción tuvo mayor impacto en el comercio. Por lo tanto, la clave para aumentar los flujos comerciales es desarrollar la infraestructura y la capacidad de los países de establecer servicios de entrega eficaces y reducir el precio de los productos transados.

\section{Otros resultados: importancia de las contrapar- tes comerciales y de la infraestructura de los países informantes}

Para analizar por separado el papel que desempeñan la infraestructura de los países informantes y la de los socios comerciales, se construyó un tercer modelo de gravedad, utilizando el mismo marco teórico. La diferencia con los anteriores es que contempla dos variables de la distancia geográfica en vez de una sola: la distancia geográfica del país informante (los cinco miembros de la Comunidad Andina) y la de su contraparte.

Los resultados obtenidos figuran en el cuadro 5. Los coeficientes que corresponden al tamaño económico y las variables ficticias para $A C P$ y frontera, respectivamente, evolucionan más o menos de la misma manera, tienen exactamente el mismo signo y se encuentran aproximadamente al mismo nivel. El tamaño de la economía sigue influyendo positivamente en el comercio. En el mismo sentido, la variable ficticia para la frontera común acusó valores importantes y representativos hasta 1992 , antes de que cobrara impulso el acuerdo de comercio preferencial.

Pacto Andino, 1985-1995: Segundo modelo de gravedad incluida la infraestructura (de país informante y contraparte). Resultados empíricos

(En coeficientes estandarizados)

\begin{tabular}{|c|c|c|c|c|c|c|c|}
\hline Año & $\operatorname{En} Y_{i} Y_{j}$ & $\begin{array}{l}\text { En dist. geog. } \\
\text { del informante }\end{array}$ & $\begin{array}{l}\text { En dist. geog. } \\
\text { de contraparte }\end{array}$ & $\begin{array}{l}\text { Variable ficticia } \\
\text { ACP }\end{array}$ & $\begin{array}{c}\text { Variable ficticia } \\
\text { Border }\end{array}$ & $\mathrm{R}^{2}$ & $\mathrm{~N}^{\mathrm{o}}$ \\
\hline 1985 & $0,782 *$ & $-0,207^{*}$ & $-0,179^{*}$ & $-0,034$ & $0,374 *$ & 0,687 & 125 \\
\hline 1986 & $0,775^{*}$ & $-0,278^{*}$ & $-0,161^{*}$ & $-0,087$ & $0,337 *$ & 0,695 & 129 \\
\hline 1987 & $0,798 *$ & $-0,243^{*}$ & $-0,182^{*}$ & $-0,017$ & $0,327^{*}$ & 0,688 & 131 \\
\hline 1988 & $0,843^{*}$ & $-0,302 *$ & $-0,127^{*}$ & $-0,031$ & $0,328 *$ & 0,764 & 134 \\
\hline 1989 & $0,802^{*}$ & $-0,338^{*}$ & $-0,135^{*}$ & $-0,010$ & $0,302^{*}$ & 0,704 & 133 \\
\hline 1991 & $0,841^{*}$ & $-0,262 *$ & $-0,142^{*}$ & $0,175^{*}$ & $0,295^{*}$ & 0,765 & 132 \\
\hline 1992 & $0,825^{*}$ & $-0,215^{*}$ & $-0,196^{*}$ & $0,118 *$ & $0,330 *$ & 0,777 & 135 \\
\hline 1993 & $0,806^{*}$ & $-0,199 *$ & $-0,187^{*}$ & $0,154 *$ & $0,301 *$ & 0,795 & 143 \\
\hline 1994 & $0,770 *$ & $-0,191^{*}$ & $-0,203^{*}$ & $0,190 *$ & $0,271 *$ & 0,747 & 146 \\
\hline 1995 & $0,750^{*}$ & $-0,227 *$ & $-0,211^{*}$ & $0,190 *$ & $0,220 *$ & 0,729 & 151 \\
\hline
\end{tabular}

Fuente: estimaciones de los autores.

* Significativo al $5 \%$. 
El cuadro 5 muestra también que, hasta 1992, la infraestructura de los países informantes, esto es, los mismos miembros de la Comunidad Andina, influyó más negativamente en el comercio que la de la contraparte en el comercio bilateral. Ello indica claramente que a fines del decenio de 1980 y principios del de 1990 , la falta de infraestructura y la consiguiente desventaja respecto de otros países de la región afectaron negativamente las oportunidades de comercio de los miembros del Pacto. Al igual que en muchos países de América Latina, su infraestructura sufrió un deterioro significativo en los años 1980 y principios de los 1990, período en que la región perdió apreciable terreno en relación con los países industrializados y las economías emergentes de crecimiento más acelerado (Calderón y Servén, 2003). Los coeficientes revelan que la falta de infraestructura de los países informantes contribuyó a reducir las posibilidades de comercio en mayor medida que la de su contraparte. En 1989 y 1990, los efectos de la infraestructura del país informante fueron aproximadamente dos y media veces más negativos que los de la contraparte.

Cuando la infraestructura empezó a adquirir importancia dentro de las metas de los gobiernos, los costos del transporte se redujeron y pudieron alcanzarse destinos distintos y más lejanos a precios similares. Aumentó la importancia de la infraestructura de la contraparte comercial mientras que la del país informante se redujo. Los resultados indican que el gran esfuerzo realizado por los países andinos para acrecentar la participación del sector privado en el desarrollo de la infraestructura fue fructífero. Además, a partir de 1990 aumentó el valor absoluto de la elasticidad de la distancia. Se logró reducir la escasez de financiamiento con cargo a recursos públicos y mejorar la productividad de la infraestructura (Estache, Wodon y Foster, 2002), con lo cual la distancia volvió a tornarse más flexible. En 1995, la infraestructura de los países informantes y la de sus contrapartes adquirió importancia en la reducción de costos y como factor determinante de la eficiencia.

\section{Perspectivas de la infraestructura regional en la Comunidad Andina}

La infraestructura no solo debería utilizarse como mecanismo clave para la integración, sino también como un vínculo con el desarrollo sostenible. A continuación, nos referimos brevemente a la situación en que se encuentra la infraestructura en la región andina. Refiriéndonos a las características de los corredores existentes y esbozando la situación de los que parecen tener mejores posibilidades de desarrollo, tratamos de vincular nuestras conclusiones con la situación real. En efecto, para fomentar el comercio intrarregional y la integración física, los miembros de la Comunidad han comenzado a adoptar medidas comunes en varios frentes orientadas a facilitar y desregular los servicios de transporte, el suministro de energía y las telecomunicaciones. ${ }^{12} \mathrm{Se}$ adoptaron medidas concretas respecto de todos los medios de transporte, incluido el transporte multimodal, para establecer los principios y criterios necesarios para proporcionar servicios eficientes. ${ }^{13}$

$\mathrm{Al}$ creciente comercio intrarregional de la Comunidad Andina, examinado en los párrafos anteriores, se sumó la concentración de los mercados. El par de países que tiene mayor participación en el comercio intrarregional es el formado por Colombia-República Bolivariana de Venezuela, mientras que el de Colombia-Ecuador se sitúa en segundo lugar.

Según el BID (2000), en Sudamérica los flujos comerciales se concentran en torno a un número reducido de corredores y centros de actividad conexos, pero solo uno de los seis principales se encuentra en la Comunidad Andina. Los flujos más importantes no corresponden a la Comunidad, sino al Cono Sur, en que Brasil, Chile y, hasta 2001, Argentina ocuparon los principales lugares (cuadro 6). Con todo, el centro Colombia-Venezuela, que conecta a Bogotá y Caracas, mueve más de 3 millones de toneladas anuales de carga y lo supera únicamente el de ArgentinaBrasil. La mitad de esta carga, que en 1998 ascendió a 2.577,8 millones de dólares, se transporta en camión y el resto por transporte fluvial y marítimo. También existe una línea de transmisión eléctrica de 380 MW de capacidad. La corriente de comercio entre Ecuador y Colombia, que en 1998 ascendió a 856,5 millones de dólares, ocupa el noveno lugar en importancia. El comercio intrarregional está aumentando gradualmente. En 2002, cerca de 50\% de los bienes comerciados fueron productos de alto valor agregado, y en el 50\% restante, de bajo valor agregado, se destaca el petróleo.

Ya se examinaron los distintos problemas conceptuales, marcos de análisis y medidas orientadas a desarrollar la infraestructura regional que se están aplicando en la

\footnotetext{
12 www.comunidadandina.org/servicios/trans.htm.

${ }^{13}$ En el campo del transporte terrestre, por ejemplo, las Decisiones 398 (pasajeros) y 399 (mercancías) establecieron las condiciones contractuales y las responsabilidades del transportista y del usuario, y mediante la Decisión 467 se reglamentó el transporte internacional por carreteras. La Resolución 300 reguló la Decisión 399, estableciendo los formularios que deben utilizar las autoridades y los transportistas. Asimismo, en el caso del transporte marítimo y del transporte aéreo se tomaron importantes medidas orientadas a uniformar las políticas y a aumentar la competitividad de las empresas.
} 
CUADRO 6

Sudamérica: diez principales relaciones de comercio bilateral, 1998

(En millones de dólares)

\begin{tabular}{lrr}
\hline Asociados en comercio bilateral & Flujos & $\%$ \\
\hline Argentina-Brasil & 14411,3 & 38,64 \\
Colombia-Venezuela (Rep. Bol. de) & 2577,8 & 6,91 \\
Argentina-Chile & 2413,5 & 6,47 \\
Brasil-Chile & 1851,0 & 4,96 \\
Brasil-Uruguay & 1815,6 & 4,87 \\
Brasil-Paraguay & 1598,7 & 4,29 \\
Brasil-Venezuela (Rep. Bol. de) & 1367,3 & 3,67 \\
Argentina-Uruguay & 1338,1 & 3,59 \\
Colombia-Ecuador & 856,5 & 2,30 \\
Argentina-Paraguay & 751,7 & 2,02 \\
\hline
\end{tabular}

Fuente: Banco Interamericano de Desarrollo (2000).

Comunidad Andina. Sin embargo, en la búsqueda de una visión estratégica común para alcanzar el desarrollo que sea válida no solo en la región andina, sino para toda América Latina, la Comunidad se incorporó a la iniciativa para la Integración de la Infraestructura Regional de Sudamérica (IIRSA).

La IIRSA es un concepto político y estratégico regional basado en el desarrollo de un eje de integración que comprende los 12 países de Sudamérica. Representa una nueva forma de abordar las tareas de planificación, coordinando tanto las políticas sectoriales nacionales como la realización de proyectos que sean compatibles con las políticas de los asociados regionales. Por lo tanto, al analizar los corredores posibles habría que tener presente aquellos en que participan los países andinos como integrantes de la Comunidad Andina $y$ también los que comunican con los demás socios comerciales actuales y potenciales de América Latina.

Los centros de intercambio que canalizan los flujos principales se complementan con otros en que el volu-

\section{VI}

\section{Conclusiones}

En el presente trabajo se estudiaron tres modelos de gravedad diferentes, pero relacionados. El primero comprobó la importancia del acuerdo de comercio preferencial andino y de la proximidad geográfica en las corrientes comerciales de sus miembros. El segundo y el tercero también tuvieron presente la evolución del acuerdo, así como los aspectos relacionados con la men transado es menor, pero que tienen un importante potencial de crecimiento. Estos corredores de menor importancia en términos de volumen son precisamente aquellos en que nuevas inversiones pueden generar más rentabilidad al reducir los estrangulamientos y ampliar la capacidad. El hecho de abordar el regionalismo, a partir de un conjunto de centros de actividad y de corredores, permite identificar flujos que podrían estimularse promoviendo la integración en distintos campos, aprovechando las complementariedades entre las distintas economías $\mathrm{y}$ creando planes orientados a vincular a otras regiones con las redes existentes. Este enfoque apunta a convertir los centros de actividad comercial en ejes de integración y desarrollo en que la infraestructura no se considera en forma aislada, sino como parte de un conjunto de actividades, vinculando -mediante distintos tipos de integración- las inversiones físicas con los aspectos sociales del desarrollo.

Los resultados que hemos obtenido confirman la importancia de lo antes señalado y constituyen un estímulo adicional para emprender este tipo de actividades. Sin embargo, existen varias alternativas para las inversiones en infraestructura: desde las de tipo local o interno (el "costo país") a las que favorecen a los socios situados a mayor distancia. Desde el punto de vista del desarrollo regional, las más adecuadas son las primeras y las vinculadas con la IIRSA, sea dentro o fuera de la Comunidad.

El funcionamiento de nuevas zonas de libre comercio en la región, tales como la de Mercosur-Comunidad Andina, podrían cambiar el mapa comercial de Sudamérica. La información proporcionada en este trabajo indica que una de las prioridades que deberían tenerse presentes en este tipo de acuerdos es el desarrollo de los centros de actividad y corredores pertinentes. proximidad, pero incluyeron el papel que desempeña la infraestructura. Uno de los modelos evaluó la importancia general que tiene acortar la distancia entre los socios en el comercio bilateral, y el otro separó los efectos de la dotación de infraestructura por importador y exportador, a fin de determinar cuál es más importante para acortar la distancia física. 
Todos los resultados confirmaron que en materia de comercio el tamaño de la economía tiene una importancia decisiva. Cabe destacar que, incluso dentro de los acuerdos regionales, dicho tamaño define en qué medida un país determinado tiene poder de negociación. Cuando se trata de comercio, lo que interesa a los Estados es su capacidad de compra y, por lo tanto, el poder económico de los demás. Y como el tamaño de la economía no puede modificarse fácilmente mediante políticas de corto plazo, para estimular no solo el comercio, sino también el crecimiento, al comienzo los países deberían centrar la atención en variables tales como la infraestructura o los acuerdos de comercio preferencial. Aun así, toda negociación regional debe tener presente el tamaño de las economías.

El primer modelo de gravedad confirmó que la Comunidad Andina influyó favorablemente en el comercio dentro de la región y con terceros países. Tanto la evolución positiva como el bajo valor de los coeficientes implican que el acuerdo de comercio preferencial se fue fortaleciendo lentamente, debido a la complejidad y al elevado número de excepciones del proceso de integración. El segundo modelo confirmó que el acuerdo de comercio preferencial solo adquirió importancia en los años 1990, cuando los miembros pusieron en marcha la Zona de Libre Comercio. También puso de manifiesto que su impacto es mayor cuando se tiene en cuenta la dotación de infraestructura. Reduciendo el costo y mejorando la calidad de los sistemas de transporte mediante el desarrollo de la infraestructura facilita el acceso a los mercados internacionales y estimula el aumento del comercio.

A medida que en el mundo se arraiga el nuevo regionalismo (BID, 2002) y que la liberalización continúa reduciendo los aranceles y los obstáculos al comercio, el grado real de protección atribuible a los costos del transporte asociados a una infraestructura deficiente puede ser considerablemente superior al que proporcionan los aranceles. No hay duda alguna de que la Comunidad Andina debería replantear la forma de abordar el proceso de integración, poniendo en marcha mecanismos adecuados para mejorar su estabilidad geopolítica, atraer inversión extranjera directa, fomentar una cooperación regional de carácter funcional —especialmente en materia de infraestructura - y mejorar su posición negociadora a nivel económico y político frente a otros países o agrupaciones. Pero también debería fomentar un nuevo tipo de integración que, en vez de limitarse a adoptar medidas relacionadas con el comercio, se oriente hacia la cooperación en distintos frentes y hacia la competencia a nivel mundial. De lo contrario, los efectos del acuerdo de comercio preferencial se irán diluyendo gradualmente a medida que en otros países de América Latina se reduzcan los aranceles en virtud de nuevos acuerdos regionales. Con esto, el comercio bilateral dependerá en último término de los costos y de la competitividad. Sin embargo, la única forma de lograrla es mejorando los servicios logísticos y de transporte en todas las etapas de la cadena de producción y distribución, y la consiguiente reducción de los costos generada por un tipo más amplio de integración regional.

El segundo modelo también demostró que el hecho de tener una frontera común que permita el comercio fronterizo ya no influye tanto. A medida que se redujeron los costos del transporte y que se fue afirmando el acuerdo de comercio preferencial, promoviendo también el mejoramiento de la infraestructura, las fronteras fueron perdiendo importancia. Sin embargo, como la principal modalidad de transporte de gran parte del creciente flujo de mercancías es el transporte terrestre y el comercio fronterizo es importante fuente de actividad económica entre vecinos, para integrar eficazmente las economías nacionales es preciso que las fronteras interandinas cuenten con equipamiento adecuado. Esto último es fundamental para abrir corredores comerciales y centros de desarrollo que conecten a través de las fronteras las regiones interiores de los países andinos con puertos del Pacífico y el Atlántico. Estos corredores permitirán que se establezcan verdaderos cruces de caminos, cuya principal ventaja será su ubicación geográfica privilegiada.

Una vez más, la evolución, signo, significancia y valores de la variable correspondiente a la distancia geográfica pusieron de relieve la importancia de la infraestructura para el comercio. Asimismo, constituyen una fuerte señal de que, en la medida en que el Pacto Andino llegue a ser un acuerdo de integración más amplio y perfeccionado, la infraestructura es la variable más manejable con que cuentan los gobiernos para reducir los costos del transporte.

Los resultados del último modelo de gravedad, que considera por separado la infraestructura de los países andinos y la de sus contrapartes, revelan que actualmente la infraestructura de un país es un factor decisivo no solo para importar los bienes que necesita localmente, sino también para reunir los requisitos necesarios como asociado comercial. El mejoramiento de la infraestructura en los países pobres o de ingresos medianos, como los países andinos, trae consigo una elevada rentabilidad global en materia de comercio (Brun, Carrère y otros, 2002).

Por último, el mejoramiento de la infraestructura no solo debe considerarse como un mecanismo para 
aumentar el comercio en el marco de la cooperación funcional entre las economías sudamericanas, sino como un importante factor de desarrollo que permitirá

\section{APÉNDICE}

\section{El índice de la infraestructura}

Para construir un índice de la infraestructura, los autores han utilizado diferentes métodos. Owen (1987) clasificó los países en función de su infraestructura utilizando un promedio lineal de varias medidas de esta y atribuyendo un valor de 100 a un país determinado con el cual relacionó a los demás. Hulten (1996) optó por normalizar los datos individuales sobre la infraestructura en cuartiles. A continuación, asignó un valor a cada uno de ellos y a partir de esta clasificación construyó un índice con los promedios (Calderón y Chong, 2004). El índice que obtuvieron Limão y Venables (2001) se basó en cuatro variables: kilómetros de caminos, de carreteras pavimentadas y de líneas férreas por kilómetro cuadrado del territorio, y troncales telefónicas por persona. Para normalizar las variables utilizaron los componentes de los factores además de una función Cobb-Douglas de la producción. En todo caso, los autores - al igual que otros que utilizan sistemas parecidos- afirmaron que, en general, la normalización no influye en los resultados. Martinez-Zarzoso y Nowak-Lehmann (2002) utilizaron las mismas cuatro variables de la infraestructura, pero normalizaron solamente la variable relativa a líneas telefónicas para 1.000 personas, obteniendo un promedio simple para el índice de la infraestructura de cada país.

El índice que utilizamos en este trabajo se calculó sobre la base de cinco variables de la infraestructura: las cuatro utilizadas por Limão y Venables (2001) más los kilowatts de capacidad de generación eléctrica. Por lo general, las variables correspondientes a cantidad se normalizaron a fin de que no dependan del tamaño del país. Por lo tanto, las troncales telefónicas y los kilowatts de electricidad se dividieron por la población, y los caminos, carreteras pavimentadas y ferrocarriles se normalizaron por kilómetro cuadrado de superficie del país. Este procedimiento se basó en Canning, para quien era adecuado normalizar los productos competidores de acuerdo con la población, puesto que la cantidad de bienes dividida por la población indica el consumo promedio. ${ }^{14}$ Sin embargo,

\footnotetext{
${ }^{14}$ Un producto es de carácter competitivo cuando el uso que un agente hace de él excluye el uso simultáneo del mismo producto por otros agentes. (Véase "Non-rival productivity imputs", disponible en: www. hassler-j.iies.su.se/Courses/macro/2000/growth3.pdf).
}

que la región en su conjunto sea más competitiva a la vez que un socio atractivo para otras ubicaciones más distantes.

en el caso de productos que compiten, el consumo promedio per cápita no se obtiene normalizando por la población, puesto que al disponer de un determinado nivel de infraestructura no competidora el incremento de la población no reduce el consumo promedio. Por eso tiene sentido normalizar los datos sobre la infraestructura de transportes por superficie, como lo hicieron Ingram y Liu (1997), Limão y Venables (2001) y también nosotros.

Los kilowatts de electricidad se incluyeron debido a que la electricidad contribuye a la actividad económica en general, y es fundamental en las actividades relacionadas con las telecomunicaciones, la computación y la maquinaria. Además, al menos en un punto del proceso de transporte y comercio la mayoría de las actividades depende de la electricidad, por ejemplo, el funcionamiento de los puertos y el procesamiento de información. Por otra parte, la electrificación adecuada de los caminos permite el transporte seguro y eficiente de las mercancías, sobre todo de noche, que es precisamente cuando tiene lugar gran parte del transporte por carreteras en los países andinos.

Por falta de datos comparables entre países y respecto de un lapso de tiempo adecuado, excluimos la información relacionada con puertos y aeropuertos, que representan una proporción menor de la dotación de infraestructura. Por la misma razón, en materia de energía solo se incluyó la electricidad. Además, el análisis solo contempla la cantidad y no la calidad de las existencias, ya que prácticamente no hay información sobre la eficiencia de los servicios.

El índice final es un promedio lineal de las cinco variables (normalizadas) de la infraestructura, calculado para cada país de la muestra en el período 1985-1995. El valor del índice para los países respecto de los cuales se realizan las regresiones puede solicitarse a los autores. Como se dijo, al normalizar las variables de la infraestructura se eliminan los efectos del tamaño. De ahí las elevadas cifras que registran algunos países pequeños, como Bélgica, los Países Bajos y Japón, cuya infraestructura está bien desarrollada, pese a que en términos de kilómetros o de número de teléfonos parecen disponer de menos infraestructura.

(Traducido del inglés) 


\section{Bibliografía}

Anderson, J. E. y E. van Wincoop (2003): Gravity with gravitas: a solution to the border puzzle, The American Economic Review, vol. 93, $\mathrm{N}^{\circ}$ 1, Nashville, Tennessee, American Economic Association.

BID (Banco Interamericano de Desarrollo) (2000): A New Push for Integration of Regional Infrastructure, Washington, D.C. (2002): Más allá de las fronteras: el nuevo regionalismo en América Latina, Washington, D.C.

(2004): Regional Public Goods: From Theory to Practice, Washington, D.C.

Bougheas, S., P. Demetriades y E. Morgenroth (1999): Infrastructure, transport costs and trade, Journal of International Economics, vol. 47, $\mathrm{N}^{\circ} 1$, Amsterdam, Elsevier.

Brun, J.F., C. Carrère y otros (2002): Has Distance Died? Evidence from a Panel Gravity Model, CEPR Discussion Paper, $\mathrm{N}^{\circ} 3500$, Londres, Centre for Economic Policy Research (CEPR).

Calderón, C. y L. Servén (2003): The output cost of Latin America's infrastructure gap, en W. Easterly y L. Servén (comps.), The Limits of Stabilization: Infrastructure, Public Deficits, and Growth in Latin America, Stanford, Stanford University Press.

Calderón, C. y A. Chong (2004): Volume and quality of infrastructure and the distribution of income: an empirical investigation, Review of Income and Wealth, vol. 50, $\mathrm{N}^{\circ} 1$, Oxford, Reino Unido, Blackwell Publishing.

Carrillo, C. y C.A. Li (2002): Trade Blocks and the Gravity Model: Evidence from Latin American Countries, Working Paper, Essex, Colchester, Reino Unido, Universidad de Essex.

Comunidad Andina (s/f): Flujos comerciales intercomunitarios por modos de transporte, 1997-1999. Disponible en www.comunidadandina.org.

Echavarría, J.J. (1998): Flujos comerciales en los países andinos: ¿liberalización o preferencias regionales? Coyuntura económica, vol. 28, №3, Bogotá, D.C., Fundación para la Educación Superior y el Desarrollo (FEDESARROLLO)

Estache, A., Q. Wodon y V. Foster (2002): Accounting for Poverty in Infrastructure Reform: Learning from Latin America's Experience, Washington, D.C., Banco Mundial.
FMI (Fondo Monetario Internacional) (2001): Directory of Trade Statistics Yearbook, Washington, D.C.

Frankel, J. (1997): Regional Trading Blocs in the World Economic System, Washington, D.C., Instituto de Economía Internacional.

Hulten, C.R. (1996): Infrastructure Capital and Economic Growth: How Well You Use It May Be More Important Than How Much You Have, NBER Working Paper, $\mathrm{N}^{\circ}$ 5847, Cambridge, Massachusetts.

Hummels, D. (1998): Toward a Geography of Transport Costs, Chicago, Universidad de Chicago, inédito.

(1999): Have International Transport Costs Declined?, Chicago, Universidad de Chicago, inédito.

Ingram, G. y Z. Liu (1997): Motorization and the Provision of Roads in Countries and Cities, Research Working Paper, $\mathrm{N}^{\circ} 1842$, Washington, D.C., Banco Mundial.

Krugman, P. (1991): The move toward free trade zones, Policy Implications of Trade and Currency Zones, Kansas City, Federal Reserve Bank of Kansas City.

Limão, N. y A. Venables (2001): Infrastructure, geographical disadvantage, transport costs and trade, World Bank Economic Review, vol. 15, $\mathrm{N}^{\circ} 3$, Washington, D.C., Banco Mundial.

Martinez-Zarzoso, I. y F. Nowak-Lehmann (2002): Explaining Mercosur Sectoral Exports to the EU: The Role of Economic and Geographical Distance, Working Paper $\mathrm{N}^{\circ} 85$, Goettingen, Ibero-American Institute for Economic Research (IAI), Universidad de Goettingen.

Owen, W. (1987): Transportation and World Development, Baltimore, The Johns Hopkins University Press.

Porojan, A. (2000): Trade Flows and Spatial Effects: The Gravity Model Revisited, Discussion Paper, $\mathrm{N}^{\circ}$ 00/04, Exeter, University of Exeter, School of Business and Economics.

Summers, R. y A. Heston (1991): The Penn world tables (mark 5): an expanded set of international comparisons 1950-1988, Quarterly Journal of Economics, vol. 106, $\mathrm{N}^{\circ}$ 2, Cambridge, Massachusetts, The MIT Press.

Wilson, J. (2003): Trade facilitation, WTO rules, and capacity building: What's at stake?, Development Outreach, vol. $5, \mathrm{~N}^{\circ} 2$, Washington, D.C., World Bank Development Institute. 